\title{
Liquid Crystals and Phase Equilibria Binary Bile Salt-Water Systems
}

\author{
Eduardo F. Marques, ${ }^{*,+, \neq}$ Håkan Edlund, ${ }^{\S}$ Camillo La Mesa," and Ali Khan ${ }^{\dagger}$
}

Physical Chemistry 1, Center for Chemistry and Chemical Engineering, P.O. Box 124, Lund University, Lund SE - 221 00, Sweden; Departamento de Química, Universidade de Coimbra, 3049 Coimbra, Portugal; Department of Chemistry and Process Technology, Chemistry, Mid Sweden University, SE -851 70 Sundsvall, Sweden; and Dipartamento de Química, Università degli Study “La Sapienza”, p.le Aldo Moro 5, 00185 Roma, Italy

Received September 28, 1999. In Final Form: February 16, 2000

\begin{abstract}
The phase behavior of several binary sodium bile salt-water systems is investigated over the entire concentration range, with emphasis on concentrated regions beyond the isotropic solution phase. The studied bile acid salts comprise the free salt sodium deoxycholate (SDC), the taurine conjugates sodium taurocholate (STC), sodi um taurodeoxycholate(STDC), and sodium taurochenodeoxycholate(STCDC) and the glycine conjugate sodium glycodeoxycholate (SGDC). A combination of classical techniques is used, including phasediagram determination, polarizing microscopy, ${ }^{2} \mathrm{H} N \mathrm{NR}$, and small-angleX-ray scattering (SAXS). The aggregation behavior in the isotropi cmicellar solutions of STC and STDC is also investigated by pulsed-field gradient NMR self-diffusion. The optical textures and the data from SAXS and ${ }^{2} \mathrm{H}$ NMR clearly point to the formation of hexagonal liquid crystals, possibly of the reversetype, beyond the micellar solution for all the bile salts. Several unusual kinetic effects, such as very slow equilibration times and the formation of transient spherulitic crystals in bi phasic regions, are observed. The phase diagrams and structural data arequal itatively discussed in terms of themolecular structureand solubility of the different salts. The formation of Iyotropic liquid crystals by bile salts, which has remained unknown for decades, is clearly demonstrated in this work.
\end{abstract}

\section{Introduction}

The bileacid salts, or simply bilesalts, are amphiphilic molecules of a different kind. They are comparable to common surfactants in that they are surface-active and self-associate in water into micellar-like aggregates. ${ }^{1-3}$ Nonethel ess, their association is of low cooperativity, and this is manifested by relatively poorly defined critical micelleconcentrations (cmcs). Such amphiphilicproperties are enough to render them a fundamental role in the human body, ${ }^{4-6}$ where they occur in the bile produced by the liver and stored in the gallbladder. They act as a solubilizer for lecithin and cholesterol, forming mixed micelles with these otherwise insoluble lipids and thus assisting their excretion from the body. Not least, they form mixed micelles with fatty acids and monoglycerides, the two water-insoluble main products of fat digestion, and promote their absorption by the membrane of intestinal cells.

The peculiarity of bile salts as surface-active agents is directly linked to their molecular structure. The latter differs from that of common surfactants, for which there is a clear-cut separation between the polar (hydrophilic headgroup) and nonpol ar regions (typically, flexiblealkyl chains). The bile salts are derived from cholic acid, and so, their hydrophobic part consists of a rigid, nonplanar,

\footnotetext{
* E-mail: eduardo.marques@fkem1.lu.seor emarques@ci.uc.pt.

† Center for Chemistry and Chemical Engineering.

₹ Universidade de Coimbra.

$\S$ Mid Sweden University.

" Università degli Study “La Sapienza".

(1) Fontell, K. Kolloid-Z. Z. Polym. 1971, 244, 246.

(2) Carey, M. C.; Small, D. M. Arch. Intern. Med. 1972, 130, 506.

(3) Mysels, K. J. Hepatology 1984, 4, 80S.

(4) Carey, M. C.; Small, D. M. Am. J . Med. 1970, 49, 590.

(5) Small, D. M. The Bile Acids; Nair, P. P., Kritchevsky, D., Eds.; Plenum Press: New York, 1971.
}

(6) Borgström, B. Int. Rev. Physiol. 1977, 12, 305. steroid fused-ring system. The ring contains from one to three hydroxyl $(\mathrm{OH})$ groups, which means that small hydrophilicgroups are present in thehydrophobic moiety. In the cholic acid salt and its conjugates, the $\mathrm{OH}$ groups lie in the same side of the ring, so the latter bears both a polar and a nonpolar side (Figure 1). The rigidity and thetwo-faced character of the ring imply that thepacking of the amphiphiles into micelles or other supramolecular structures does not follow the conventional picture for surfactant aggregates. At the cmc, the bile salt molecules are believed to pack in a back-to-back way into roughly spherical micelles of small aggregation numbers, typically $\mathrm{N}_{\text {agg }}=4-10,2,7,8$ The values of $\mathrm{N}_{\text {agg }}$ increase significantly as a function of added el ectrol yte for some of the bilesalts, in accordance with typical surfactant behavior. In some systems, such as the sodium deoxycholate-water system, fibers or long rodlike structures are drawn from concentrated micellar solutions, and on the basis of X-ray data, a helical structure for the fibers has been proposed. ${ }^{9-14}$

Thestructural and interfacial properties of theisotropic solution in several binary bile salt-water systems have been studied for a few decades. $1,2,7,15-19$ There are also

(7) Mazer, N. A.; Carey, M. C.; Kwasnick, R. F.; Benedek, G. B. Biochemistry 1979, 18, 3064.

(8) Kratohvil, J. P. Adv. Colloid Interface Sci. 1986, 26, 131.

(9) Rich, A.; Blow, D. M. Nature (London) 1958, 182, 4776.

(10) Blow, D. M.; Rich, A. J . Am. Chem. Soc. 1960, 82, 3566

(11) Giglio, E.; Loreti, S.; Pavel, N. V.J . Phys. Chem. 1988, 92, 2858.

(12) Briganti, G.; D'Archivio, A. A.; Galantini, L.; Giglio, E. Langmuir 1996, 12, 1180.

(13) D'Archivio, A. A.; Galantini, L.; Giglio, E.; J over, A. Langmuir 1998, 14, 4776

(14) Bottari, E.; D'Archivio, A. A.; Festa, M. R.; Galantini, L.; Giglio, E. Langmuir 1999, 15, 2996.

(15) Ekwall, P.; Sjöblom, L. Acta Chem. Scand. 1948, 3, 1179.

(16) Ekwall, P. J . Colloid Sci. 1954, 8, 66.

(17) Murata, Y.; Sugihara, G.; Fukushima, K.; Tanaka, M.; Matsushita, K. J . Phys. Chem. 1982, 86, 4690.

(18) Kratohvil, J . P.; Hsu, W. P.; K wok, D. I. Langmuir 1986, 2, 256.

(19) Bottari, E.; Festa, M. R. Langmuir 1996, 12, 1777. 
a

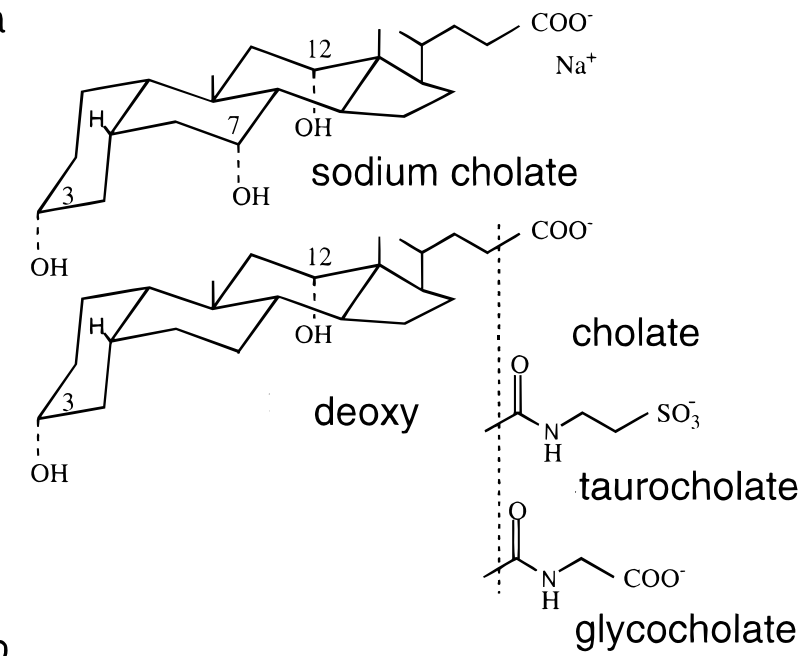

b

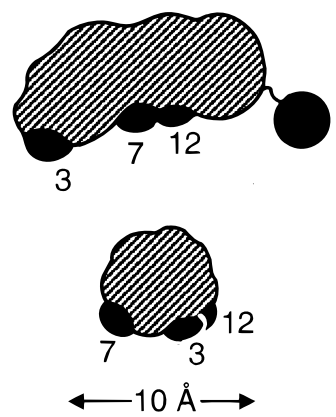

Figure 1. Molecular structure of the bile salts. (a) Sodium cholate ( $3 \alpha, 7 \alpha, 12 \alpha$-trihydroxy-5 $\beta$-cholan-24-oic-acid salt) and its deoxycholate derivative, the $3 \alpha, 12 \alpha$-dihydroxy salt. The chenodeoxy derivativeisthe $3 \alpha, 7 \alpha$-dihydroxy salt. Thestructure of the taurine and glycine conjugates is also shown. (b) Longitudinal and transverseview of thechol atemolecule, where the relative position of the $3 \alpha, 7 \alpha$, and $12 \alpha \mathrm{OH}$ groups is visible (adapted from Carey and Small.2)

reports on the phase behavior and aggregate structure of mixed systems composed of bile salt-phospholipids 20,21 and bile salt-single-chained surfactants. ${ }^{22-25}$ While bile salts can be incorporated in a variety of liquid-crystalline (Ic) phases in mixed systems, it has been assumed since early studies that they al one do not form liquid-crystalline phases in water. This observation has been acknowledged by most authors as another example of the peculiarity of bile salts. Nonetheless, in a recent report by us, it has been clearly shown by a combination of techniques that, at room temperature, sodium taurodeoxycholate (STDC) forms a wide liquid-crystalline region of hexagonal-like structure. ${ }^{26}$ It thus appears that the phase behavior of neat bilesalts in water, at concentrations higher than the micellar regions, has not been thoroughly investigated. The motivation behind this paper is thus to investigate whether theobservation in theSTDC system is a singular occurrence or, as can be reasonably expected, if a general pattern of lyotropic liquid-crystal behavior is to be found for bile salts.

(20) UImius, J .; Lindblom, G.; Wennerström, H.: J ohansson, L. B.Å.; Fontell, K.; Söderman, O.; Arvidson, G. Biochemistry 1982, 21, 1553. (21) Thurmond, R. L.; Lindblom, G.; Brown, M. F. Biophys. J . 1991, 60, 728. 327.

(22) Barry, B. W.; Gray, G. M. T. J . Colloid Interface Sci. 1975, 52,

(23) Svärd, M.; Schurtenberger, P.; F ontell, K.;J önsson, B.; Lindman, B. J. Phys. Chem. 1988, 92, 2261.

(24) Wu, K.; McGown, L. B. J . Phys. Chem. 1994, 98, 1185.

(25) Swanson-Vethamuthu, M.; Almgren, M.; Bergenståhl, B.; Mukhtar, E. J . Colloid Interface Sci. 1996, 178, 538.

(26) Edlund, H.; Khan, A.; Mesa, C. L. Langmuir 1998, 14, 3691.
Human bile is mainly composed of the sodium and potassium salts of the glycine and taurine conjugates of cholic acid and its deoxy and chenodeoxy derivatives. In this paper, we investigate the phase behavior in water of several common bilesalts, comprising the sodium salts of thefreedeoxycholicacid and some of its taurineand glycine conjugate salts (taurocholate, taurodeoxycholate, taurochenodeoxycholate, and glycodeoxycholate). The phase diagrams and the phase structure were obtained by a combination of ocular observations, polarizing microscopy, NMR methods $\left({ }^{2} \mathrm{H}\right.$ quadrupolar splitting and ${ }^{1} \mathrm{H}$ selfdiffusion), and small-angle X-ray scattering data. Slow kinetics of liquid-crystal formation and phaseequilibration seem to bethe general rulefor the binary systems at high bileconcentrations. Theassignment of the phases present in the bi phasicregions found in somesystems is not al ways straightforward. In a sense, the polymor phic character of the bile salts is also an elusive one. It is perhaps this character that has led some authors in the past to circumvent their lyotropic liquid-crystal formation.

\section{Experimental Section}

2.1. Materials. Thebileacids in the form of sodium salts were purchased from Sigma and used without further purification. $\mathrm{D}_{2} \mathrm{O}$ supplied by Dr. Basel, Switzerland, was used in all the samples for the ${ }^{2} \mathrm{H}$ and self-diffusion NMR measurements. The following bile salts were used: sodium deoxycholate (SDC), monohydrate form, >99\%; sodium taurocholate (STC), >97\%; sodium taurodeoxycholate, $0.5 \mathrm{~mol} \mathrm{H}_{2} \mathrm{O} / \mathrm{mol}$, >97\%; sodium taurochenodeoxycholate (STCDC), high purity; and sodium glycodeoxycholate (SGDC), $1.5 \mathrm{~mol} \mathrm{H}{ }_{2} \mathrm{O} / \mathrm{mol}$, >98\%. The molecular structure for sodium cholate (the free acid form) can be seen in Figure la, together with that of the deoxy derivative of the tauro and glyco conjugates. The cholic acid salt has hydroxyl groups in the three carbon positions $\alpha \mathrm{C}-3, \alpha \mathrm{C}-7$, and $\alpha \mathrm{C}-12$ (Figure 1a). These three hydroxyl groups and the two hydroxyl groups in the deoxy derivative $(\alpha \mathrm{C}-3$ and $\alpha \mathrm{C}-12)$ point to the same side of thesteroid ring, giving it one polar and onenonpolar side. The chenodeoxy derivative differs from the deoxy one in that the two $\mathrm{OH}$ groups lie in positions $\alpha \mathrm{C}-3$ and $\alpha \mathrm{C}-7$. The relative spatial position of thethreecarbon atoms is more easily visualized in Figure $1 b$.

2.2. Sample Preparation and Phase Diagram Determination. Thesamples were prepared by weight in test tubes which were flame-sealed and thoroughly mixed by repeated centrifugation. They werethen allowed to stand at the desired temperature in an oven for equilibration over several weeks. Preliminary studies of the phase behavior involved the inspection of all samples between crossed polaroids in order to check for birefringence. Further assignment of the phase behavior was based on the combined experimental data supplied by polarizing microscopy, ${ }^{2} \mathrm{H}$ NMR, and SAXS. Thesametechniques were used to delineate phase boundaries.

2.3. Polarized Light Microscopy. Under polarized light, isotropic phases show as a dark background in the microscope, whileanisotropic phases show characteristic optical textures. ${ }^{27,28}$ An Axioplan Universal polarizing light microscope (Carl Zeiss), equipped with a highly sensitive SIT C video-camera system and Argus-20 image processor (Hamamatsu Photonics, J apan), was used. The microscope is also equipped with a hot stage operating between -20 and $+100^{\circ} \mathrm{C}$.

2.4. Small-Angle $X$-ray Scattering (SAXS). The measurements were carried out in a K ratky compact small-angle system equipped with a position-sensitive detector, containing 1024 channel s of width $53 \mu \mathrm{m}$. A Cu K $\alpha$ radiation of wavelength 1.542 $\AA$ was used, and the sample-to-detector distance was $277 \mathrm{~mm}$. The samples for SAXS were prepared in a paste hol der between thin mica windows, and thetemperaturein thesamplecontainer was regulated by a Peltier element (accuracy within $\pm 0.1^{\circ} \mathrm{C}$ ). The camera volume was kept under vacuum to minimize air scattering.

(27) Rosevear, F. B. J . Am. Oil Chem. Soc. 1954, 31, 628.

(28) Rosevear, F. B. J . Soc. Cosmet. Chem. 1968, 19, 581. 
2.5. ${ }^{2} \mathrm{H}$ (Deuterium) Quadrupolar Splitting NMR. The method was carried out by using deuterated water $\left(D_{2} O\right)$ in the samples. The ${ }^{2} \mathrm{H}$ nucleus has a spin quantum number $\mathrm{I}=1$, and therefore, it possesses an electric quadrupole moment. In anisotropicliquid-crystallinephases (e.g., hexagonal and lamellar phases), the interaction of the quadrupole moment with nearby electric field gradients gives rise to a ${ }^{2} \mathrm{H}$ splitting. In isotropic phases (solutions and cubic phases), the interaction is averaged to zero by the rapid isotropic molecular motions, and thus, a singlesignal results. I n multiphasesamples, duetosl ow deuteron exchange between the phases, the resultant ${ }^{2} \mathrm{H}$ spectrum is a superposition of the spectra of the individual phases. The technique is thus effective in delineating multiphase regions, involving two or more liquid-crystalline phases (often difficult to macroscopically separate) or small amounts of isotropic phases. ${ }^{29-31}$ The magnitude of the ${ }^{2} \mathrm{H}$ quadrupolar splittings $(\Delta)$, measured as peak-to-peak separations in hertz, depends on the degree of anisotropy of the phase. For a nonoriented liquidcrystalline sample, $\Delta$ is given by

$$
\Delta=\frac{3}{4} \sum \mathrm{p}_{\mathrm{i}} \vartheta_{\mathrm{Q}}^{\mathrm{i}} \mathrm{S}_{\mathrm{i}}
$$

where $p_{i}$ is the fraction of deuterons in site $i$, characterized by the order parameter $S_{i}$ and the effective quadrupolar coupling constant $\vartheta_{0}{ }^{i}$. In surfactant-water systems, water associates to theheadgroups of theamphiphilicmolecules which areorganized into different aggregate structures. A two-site model can thus be applied with free and bound water molecules. Assuming a fast exchange between the sites and $\Delta=0$ for free water, the observed $\Delta$ for a given liquid-crystalline phase is related to the molar ratio between surfactant and water through

$$
\Delta=\left|\vartheta_{\mathrm{Q}} \mathrm{S}\right| \mathrm{n} \frac{\mathrm{X}_{\mathrm{S}}}{\mathrm{X}_{\mathrm{W}}}
$$

where $X_{S}$ and $X_{W}$ are, respectively, the molar fractions of surfactant and water, $\mathrm{n}$ is the average hydration number of the surfactant, and $\vartheta_{\mathrm{Q}}$ is the deuteron quadrupolar coupling constant.

The ${ }^{2} \mathrm{H}$ spectra were recorded at a resonance frequency of $41.45 \mathrm{MHz}$ in a J EOL FX 270 superconducting pulsed FT NMR spectrometer. A variable-temperature control unit was used to regulate the temperature of the air flow in which the samples werelocated. After being rapidly transferred tothethermostated NMR probe, the samples were kept there for a sufficiently long period to avoid a temperature gradient before the spectra were recorded. The recordings were repeated over several weeks until no changes in the pattern of the spectra were observed.

2.6. Pulsed Field Gradient (PFG) NMR Self-Diffusion. The PFG technique for measuring self-diffusion coefficients is based on a combination of a sequence of radio frequency ( $\mathrm{rf}$ ) pulses and magnetic field gradient pulses. ${ }^{32,33}$ In this method, one monitors the attenuation of a ${ }^{1} \mathrm{H}$ spin echo resulting from the dephasing of the nuclear spins due to the combined effect of translational motion of the spins and the imposition of the gradient pulses. The self-diffusion coefficient of the diffusing spins can thus be directly extracted from the echo attenuation. The typical PFG sequence is based on the Hahn spin echo and is known as the Stejskal-Tanner experiment. It consists of two rf pulses (a $90^{\circ}$ and $180^{\circ}$ pulse) and two gradient pulses with time duration $\delta$ and separation $\Delta$ between their leading edges, placed on either side of the $180^{\circ} \mathrm{rf}$ pulse. In brief, the diffusion coefficient is determined by measuring the intensity decay of a Fourier transform starting at the center of the echo, the echo attenuation, as a function of the strength $(\mathrm{g})$ or duration $(\delta)$ of the gradient pulse. With a few approximations, the echo

(29) Khan, A.; Fontell, K.; Lindblom, G.; Lindman, B. J . Phys Chem. 1982, 86, 4266 .

(30) Marques, E.; Khan, A.; Miguel, M. G.; Lindman, B. J . Phys. Chem. 1993, 97, 4729

(31) Lindman, B.; Olsson, U.; Söderman, O. Surfactant solutions: aggregation phenomena and microheterogeneity. In Dynamics of Solutions and Fluid Mixtures by NMR; Del puech,J .-J ., Ed.; J ohn Wiley \& Sons: New York, 1995

(32) Stilbs, P. Prog. Nudl. Magn. Reson. Spectrosc. 1987, 19, 1.

(33) Price, W. S. Concepts Magn. Res. 1997, 9, 299. attenuation can be described by

$$
I(k)=I_{0} e^{-k D}
$$

where $\mathrm{I}_{\mathrm{o}}$ is the echo intensity in the absence of gradient; $\mathrm{k}=$ $(\gamma \delta \mathrm{g})^{2}(\Delta-\delta / 3)$, where $\gamma$ is the proton magnetogyric ratio; $\mathrm{D}$ is theself-diffusion coefficient; and $g, \delta$, and $\Delta$ areas defined above. In the stimulated echo sequence, which is used alternatively to the Hahn echo in spin systems with short $\mathrm{T}_{2}$ values, three $90^{\circ}$ rf pulses are used, and the field gradient pulses are switched on before the second and after the third rf pulse. 32,33

The measurements were done in a Bruker DMX200 spectrometer, operating at a ${ }^{1} \mathrm{H}$ resonance frequency of $200 \mathrm{MHz}$, with a gradient probe providing a maximum field gradient of 9 $\mathrm{T} / \mathrm{m}$. F or the monitoring of the water self-diffusion, the basic Hahn echo pulsesequencewas used. Thesurfactant self-diffusion was measured by the stimulated echo pulse sequence.

\section{Results and Discussion}

3.1. Phase Behavior. The phase behavior for the different bile salt-water systems was investigated and deter mined by a combination of visual inspection between crossed polaroids, polarizing microscopy, NMR methods, and SAXS data. The bile acid salts consist of the free salt SDC, the taurine conjugates STC, STDC, and STCDC, and the glycine conjugate SGDC. Both isothermal and isoplethal methods were used to delineate phase boundaries, with an accuracy not better than $2-3 \mathrm{wt} \%$. In a previous work, ${ }^{26}$ lyotropic liquid-crystal behavior was reported for the bilesalt STDC, at room temperature, and nofurther investigations havebeen carried out sincethen. In the current work, a global inspection of the phase diagrams in Figure $2 \mathrm{a}-\mathrm{e}$, for temperatures ranging between 5 and $40^{\circ} \mathrm{C}$, shows that (i) the formation of liquid crystals by bilesalts is a typical phenomena and (ii) there is a common pattern for this lyotropic phase behavior. Three single-phase regions as a function of concentration can be identified in Figures 2a-e: an isotropic (micellar) solution phase, an anisotropic liquid-crystalline phase, and a region with hydrated crystals. There are some noticeable differences between the salts, namely, in (i) the range of existence of thelcphasein the phase diagram, (ii) the phase equilibria in two-phase regions, and (iii) the kinetics of phase formation.

STC and STDC show well-defined phase boundaries, with occurrence of single- and two-phase regions (F igure $2 \mathrm{a}$ and $2 \mathrm{~b}$ ). F or STC, at roomtemperature, samples within the range of 60-70 wt \% display a monochromatic weak birefringencewhen viewed between crossed polaroids, and thus, they were assigned to the aniso Ic region (Figure 2a). The two-phase regi on between the isotropic solution and the Ic phase has some remarkable features. Some samples appear as cl oudy and aretaken to bea dispersion of the Ic phase in the isotropic solution, i.e., the "proper" two-phase region. In some other samples, there is an isotropic solution and a weakly ani sotropic upper phase, which only forms after a few days. From this phase, spherulitic crystals (with a characteristic macroscopic 4-fold symmetry) grow with time and eventually sediment at the bottom of the sample vial. Also, from the bottom phase, anisotropicneedles appear coming up intotheupper phase. This peculiar coexistence of spherulitic crystals with solution or birefringent phases, was previously reported by us for the STDC ${ }^{26}$ and is al so seen in the STC system. As will beshown bel ow, someattempts weremade to characterize structurally the spherulites by optical microscopy. In both the STC and STDC systems, there is complete disappearance of theanisotropicoptical texture at temperatures slightly below $35{ }^{\circ} \mathrm{C}$. From all the 

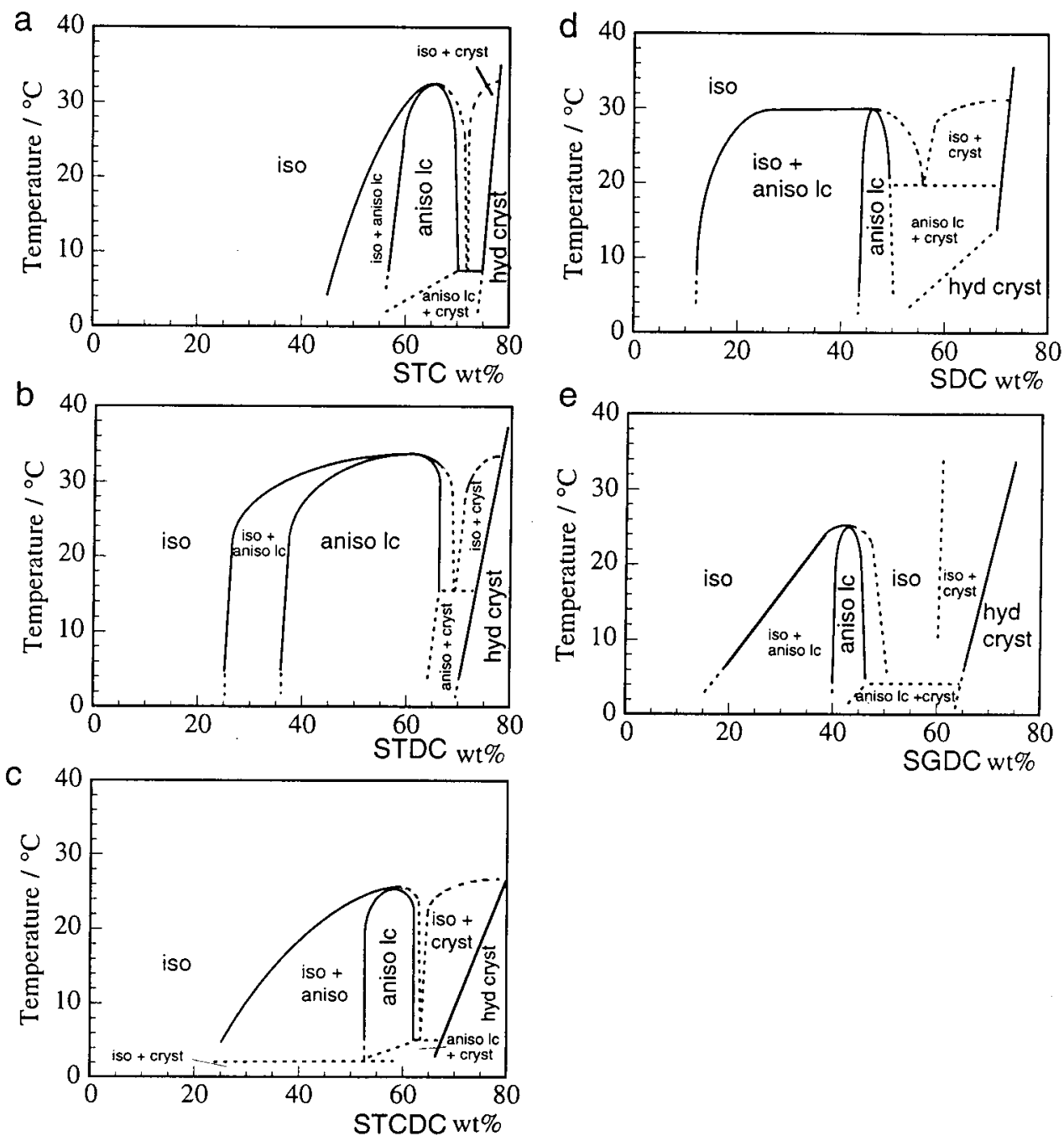

Figure 2. Phase diagrams for the different bile salt-water systems investigated here: (a) STC, (b) STDC, (c) STCDC, (d) SDC, and (e) SGDC. Notations are: iso, isotropic solution; aniso lc, anisotropic liquid crystal; cryst, crystals; and hyd cryst, hydrated crystals.

investigated systems, the STDC one shows the widest Ic phase in terms of concentration range.

The STCDC salt shows the occurrence of a narrower liquid-crystalline region than the two previous systems (Figure 2b). The upper phase-transition temperature of thephaseis about $25^{\circ} \mathrm{C}$ (F igure2C). At room temperature, a sample at 39.5 wt \% shows small spherulitic crystals on the bottom, with a weak 4-fold symmetry, while the rest of thesampleis isotropic. A sampleat $44.3 \mathrm{wt} \%$ has small spheruliti ccrystals in a cloudy anisotropic matrix. At 49.6 wt \%, no spherulitic crystals can be seen, and the sample is very cloudy. The last two samples are both transparent at visual observation and anisotropic through polarized light. At 55.5 wt \%, thesample is birefringent and gl itters, whereas a sample at 60.4 wt \% has a weaker anisotropy. This typical sequence of observations allowed us to make a preliminary delineation of phase boundaries. F or both theSTC and STCDC systems, the formation of spherulitic crystals in the biphasic region is not as clear (or even spectacular) as for STDC, but it can be seen at lower temperatures.

SDC and SGDC show a slightly more complex phase behavior. Thelcregion is quitenarrow in theconcentration range (about 5 wt \%) in both systems. A wide two-phase region between the isotropic solution and the Ic phase is present for SDC (Figure 2d). The region where optical anisotropic textures are clearly evident between crossed polaroids is al most always a two-phase region (iso/aniso or aniso/cryst) in the time span of a week. Within only a few weeks, single-phase anisotropic samples are formed. Similar observations are made for SGDC. In this system, thel iquid crystal sl owly appears from bi phasic solutioncrystal samples. It is clear that in the two systems the kinetics of liquid-crystal formation is considerably slow, and much sl ower than that for theprevious systems. Above around $32{ }^{\circ} \mathrm{C}$ for SDC and $28^{\circ} \mathrm{C}$ for SGDC, the Ic phase melts totally onto the isotropic solution.

Several relevant comments are to be made. First, the depiction of the two-phase region between the micellar solution and the liquid crystal as iso-aniso Ic in Figure $2 \mathrm{a}-\mathrm{e}$ does not convey the complete picture of the phase behavior. In view of what was described above, the formation of spherulitic crystals is a general phenomena. These structures can either be ascribed to a metastable liquid crystal or to transient nucleation sites for the formation of theanisolc phase. Second, theisotropic phase beyond the Ic phase is depicted as being identical to the isotropic micellar phase. In most systems, one finds that this isotropic viscous phase (fl owing under gravity) occurs in equilibrium with crystals. For the case of SGDC, however, it was possible to detect a single isotropic phase between the aniso Ic and the hydrated crystals. The samples areisotropicand rather stiff and clearly resemble a cubic liquid-crystalline phase more than a viscous 
a
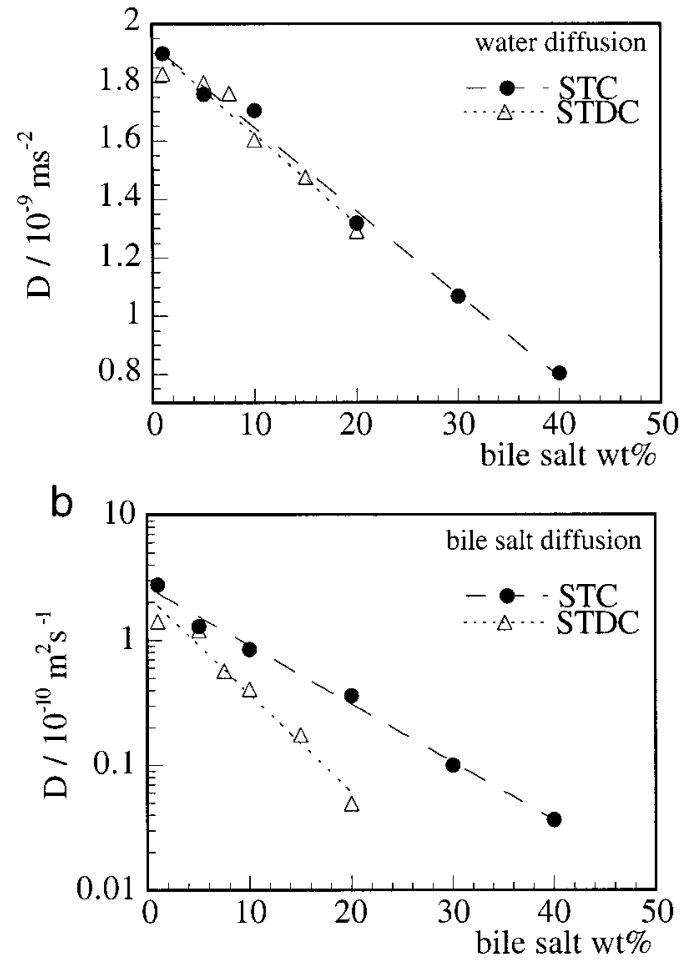

Figure 3. Self-diffusion measurements in theisotropicsolution of STC and STDC at $25^{\circ} \mathrm{C}$ : (a) water diffusion; (b) bile salt diffusion. The lines are guides for the eyes.

solution. Therefore, the possibility of there being an isotropic phasedistinct from theisotropicmicellar solution at very high surfactant concentration cannot beexcluded.

3.2. NMR Self-Diffusion Study of the Isotropic Solution Phase. A structural study of the isotropic solution of the bile sal ts was performed by means of NMR self-diffusion measurements, for the STC and STDC systems. These two molecules are representative of trihydroxy and dihydroxy salts, respectively, and allow an illustration of therelation between mol ecular structure and aggregate growth. Both the water (Figure 3a) and the bile salt (Figure $3 \mathrm{~b}$ ) diffusion were measured. It is known that the magnitude of the obstruction effect to solvent diffusion is critically dependent on thesize, shape, and concentration of aggregates. ${ }^{34}$ Thus, spherical and rodlike micelles give rise to minor obstruction effects, whereas disklike and obl ate micelles cause larger effects. In Figure 3a, nosignificant differences can beseen between the two systems in the reduction of the water diffusion coefficient (D) values as a function of concentration. The simplest interpretation for these results would bethat, as the concentration rises, a similar growth of theaggregates occurs in both systems and the aggregates should bear similar shape.

However, the surfactant diffusion shows marked differences between the two systems (Figure $3 \mathrm{~b}$ ). It must be recalled herethat at surfactant concentrations equal and above roughly $50 \times \mathrm{cmc}$, the monomer contribution to the measured surfactant diffusion coefficient is negligible. Consequently, the surfactant diffusion is virtually identical to the micellar diffusion, i.e., $D_{\text {surf }} \approx D_{\text {mic }}$. As the surfactant concentration further increases, the latter assumption is no longer valid since interaggregate obstructions must betaken into account. ${ }^{31}$ It can be seen in

(34) J önsson, B.; Wennerström, H.; Nilsson, P.-G.; Linse, P. Colloid Polym. Sci. 1986, 264, 77.
Figure $3 b$ that for STDC solutions, the $D_{\text {surf }}$ values are al ways smaller than those for STC, at the same concentration. Moreover, the decrease in $D_{\text {surf }}$ with concentration is more pronounced for STDC than for STC. A rough estimati on of the aggregate hydrodynamic radius can be madefrom the measured $D_{\text {surf }}$ values, through the simple Stokes-Einstein equation (discarding the correction for interparticleobstructions). This can bedone, for example, at 20.0 wt \%, a concentration well above the cmc of both molecules (ca. 0.08 and 0.2 wt \% for STDC and STC, respectively, at $\left.25^{\circ} \mathrm{C}\right) .{ }^{2} \mathrm{D}_{\text {surf }}$ for STDC has a value of 5.0 $\times 10^{-12} \mathrm{~m}^{2} \mathrm{~s}^{-1}$, corresponding to a hydrodynamic radius $R_{H}$ of $390 \AA$. At the same STC concentration, $D_{\text {surf }}$ is 3.6 $\times 10^{-11} \mathrm{~m}^{2} \mathrm{~s}^{-1}$ which gives $R_{H}=54 \AA$, a value 7 times smaller than that for STDC. Sincethereis a closesimilarity in structure between the two molecules, the surfactant diffusion results indicate a more pronounced aggregate growth with surfactant concentration for STDC than that for STC. As for the water self-diffusion results, a simple rationalizati on can be done. Larger aggregates for STDC, at identical bulk concentration to STC, imply a smaller concentration of aggregates. However, the solvent obstruction effect is relatively insensitive to the particle volume fraction, unless the shape of the particles is very different. ${ }^{34}$ Thus, the differences in sizeand shape between the STC and STDC micelles cannot be "sensed" by water self-diffusion, and a comparable decrease in the solvent diffusion coefficients with surfactant concentration is observed.

Thediffusion results presented herearecompatiblewith the literature reports regarding aggregation numbers for the micelles of tri- and dihydroxy bile salts and micellar growth. Mazer et al. haveshown that sodiumchol ateforms smaller micelles than the deoxy- and chenodeoxycholate derivatives. ${ }^{7}$ It has been reported that, upon addition of electrolyte $(\mathrm{NaCl})$, STDC shows a much morepronounced micellar growth than STC.2,35 The reason for these differences in micellar growth obviously liein the presence of the additional $\mathrm{OH}$ group in the trihydroxy salts. Since the dihydroxy molecule has one less hydrophilic group, the polar face of its steroid ring is more hydrophobic, and this will have large implications in the packing of the molecules. Thegain in interfacial freeenergy per molecule associated with self-aggregation is larger for the dihydroxy; therefore, from this point of view, the formation of larger micel les should befavored from start. It should be noted that the packing of di- and trihydroxy bile salts in neat and in mixed micelles with several surfactants seems to ber easonably complex and concentration-dependent. ${ }^{17}$

The packing has been viewed in terms of a preference for a perpendicular or a flat positioning with regard to the polar-apolar interface for di- and trihydroxy salts, respectively. ${ }^{7}$ Besides the hydrophobic back-to-back interactions, intermol ecular hydrogen bonding has al so been proposed to occur in the aggregate formation. ${ }^{2,17}$ It is also relevant to mention that the self-diffusion results presented herefor STC and STDC arecoherent with the phase behavior observations made in the previous section. If the STDC micelles are bigger and grow faster with concentration, then the formation of the Ic phase should occur for smaller concentrations than thosefor STC. This is indeed observed in the phase diagrams in Figure 2a,b.

3.3. Liquid-Crystal Formation. A. Polarizing Microscopy. In most surfactant systems, polarizing microscopy is invaluable in the detection of anisotropic liquid crystals and their assignment. Anisotropicoptical textures

(35) D’Archivio, A. A.; Galantini, L.; Gavuzzo, E.; Giglio, E.; Scaramuzza, L. Langmuir 1996, 12, 4660. 


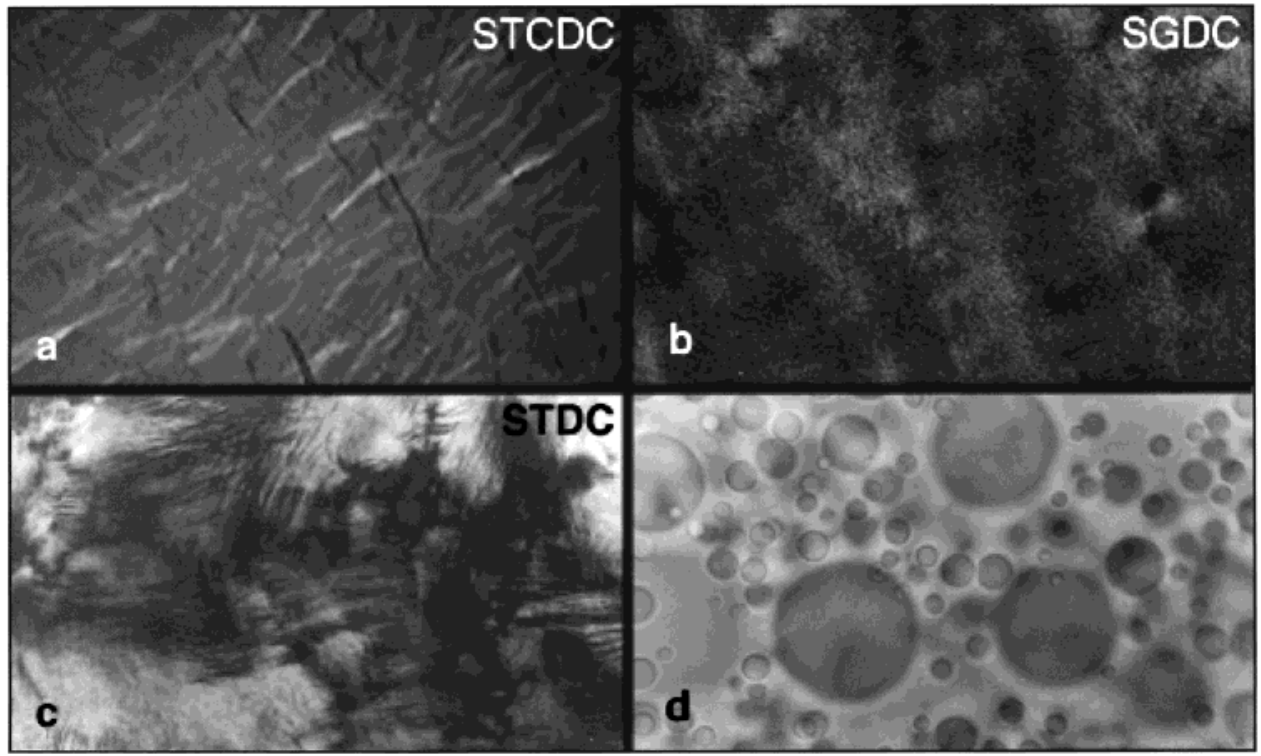

Figure 4. Liquid-crystalline textures in bile salt-water systems as seen in the polarizing microscope: (a) 57.5 wt \% STCDC, at $22^{\circ} \mathrm{C}$; (b) $42.5 \mathrm{wt} \% \mathrm{SGDC}$, at $22^{\circ} \mathrm{C}$; (c) $40.0 \mathrm{wt} \%$ STDC, at $22^{\circ} \mathrm{C}$; and (d) $40.0 \mathrm{wt} \%$ STDC, when heated to $40^{\circ} \mathrm{C}$, showing droplets in an isotropic medium (under normal light). Magnification: $200 \times$.

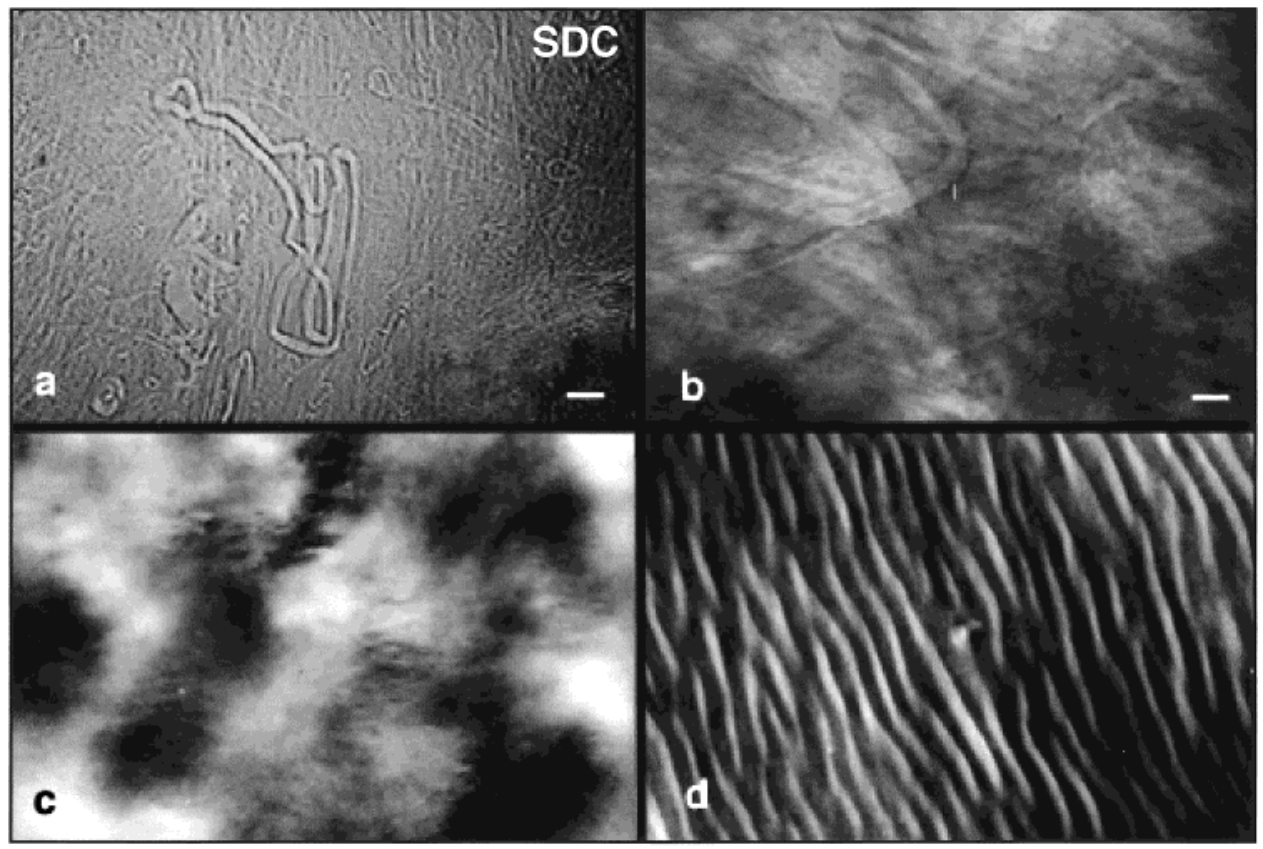

Figure 5. Polarizing microscopy observations for the SDC-water system at $22^{\circ} \mathrm{C}$ : (a) and (b) Giant fibers and anisotropic liquid-crystallinedomains at 26.6wt \%SDC, in thetwo-phase region isotropicsolution plus spherulitic crystals. (c) and (d) Ani sotropic liquid-crystalline phase for $47.1 \mathrm{wt} \%$ SDC. Magnification: $200 \times$. Bar in parts a and $\mathrm{b}$ is $40 \mu \mathrm{m}$.

were observed for the liquid-crystalline samples in all binary systems, with theexception of STC. All thetextures in Figure $4 a-c$ belong to the hexagonal type. The most well-defined textures appear for STCDC (Figure4a), where birefringent domains similar to hexagonal battonets appear, and for STDC (Figure 4c), where a clear striated nongeometric texture appears. ${ }^{27-29}$ For SGDC, a very weakly birefringent, somewhat ill-defined texture was detected (Figure 4b) of thenongeometric, nonstriated type. When STDC samples displaying the texture in Figure $4 \mathrm{C}$ at room temperature are heated above $35^{\circ} \mathrm{C}$, the liquid crystal melts, and in theglass slide, droplets of an optically isotropic phase can be seen (Figure 4d).

Attempts weremadetoseparatethespheruliticcrystals occurring in bi phasic samples and observe them between slideand cover-slipin the polarizing microscope. A sample at a composition of $26.6 \mathrm{wt} \%$ in the SDC system was selected. The bottom phase was separated from the isotropic solution and viewed in the microscope (Figure $5 a$ and $5 b)$, showing some remarkable features. Giant fiberlike structures can be seen throughout the sample (the bar in Figure 5a,b corresponds to $40 \mu \mathrm{m}$ ), forming a very densematerial. In someareas of thesample, domains of nongeometric, nonstriated textureareseen mixed with the fiberlike structures (Figure 5b). In a single-phase sample within the Ic region, at $47.1 \mathrm{wt} \%$ SDC, the anisotropic nongeometric texture is visible. Another interesting feature of this system is that by applying a small pressure onto the cover-slip, a well-defined undulating structure of thetypeimaged in Figure $5 \mathrm{~d}$ is observed. Thelong rodl ikestructures observed in Figure 5a, b appear to be in line with the helical-based hydrated fibers first 


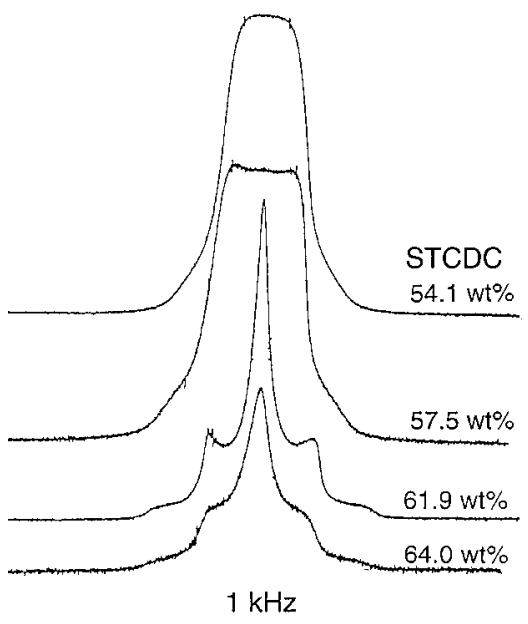

Figure 6. ${ }^{2} \mathrm{H}$ NMR spectra for the system STCDC-water, at $5^{\circ} \mathrm{C}$.

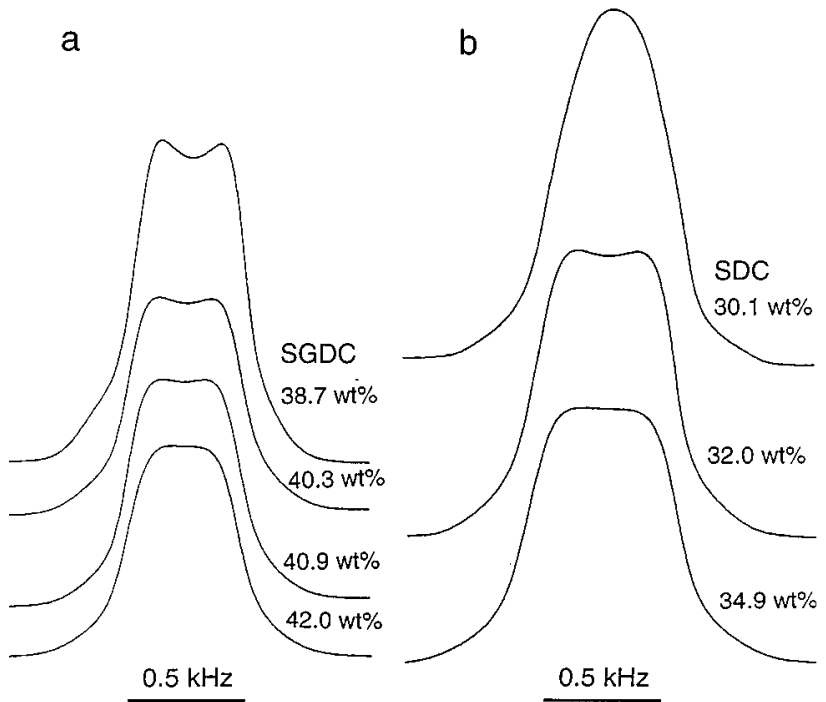

Figure 7. ${ }^{2} \mathrm{H}$ NMR spectra for the systems (a) SGDC-water and (b) SDC-water, at $7.5^{\circ} \mathrm{C}$.

reported by Bl ow and Rich a few decades ago 9,10 and more recently investigated by Gigl io et al. ${ }^{11}$ and Briganti and co-workers. ${ }^{12}$ One can speculate if the structure of the liquid-crystallinephaseis directly related with a positional and orientational arrangement of theserodlikestructures to yield anisotropy.

B. ${ }^{2} \mathrm{H}$ Quadrupolar Splitting NMR. A general observation borne out from the ${ }^{2} \mathrm{H}$ NMR study was that ${ }^{2} \mathrm{H}$ splittings could bedetected for theanisolc phasein several binary systems. Thesplitting occurs morefrequently with superi mposed isotropicsignals in the bi phasic regions. In fact, for most samples in the Ic phase of the different bile systems, thewater deuteron signal consists of a very broad signal. A representative col lection of ${ }^{2} \mathrm{H}$ spectra is shown for STCDC in Figure 6, recorded at a temperature of $5{ }^{\circ} \mathrm{C}$. At 54 wt \% of STCDC within the Ic phase, a broad signal $(\approx 0.7 \mathrm{kHz})$ is recorded, whereas at $57.5 \mathrm{w} \%$, a splitting is present. As the concentration increases toward thetwophase region, a singlet and a clear splitting are observed, indicating a two-phase region of isotropic phase and anisotropic Ic. In Figure 7a, for the SGDC system, it can be noted that a sample in the vicinity of the Ic phase boundary (38.7 wt \%) shows a splitting, while as the composition enters the Ic phase the doublet is replaced with a broad signal. For SDC (Figure 7b), a splitting could only be detected in the biphasic region.
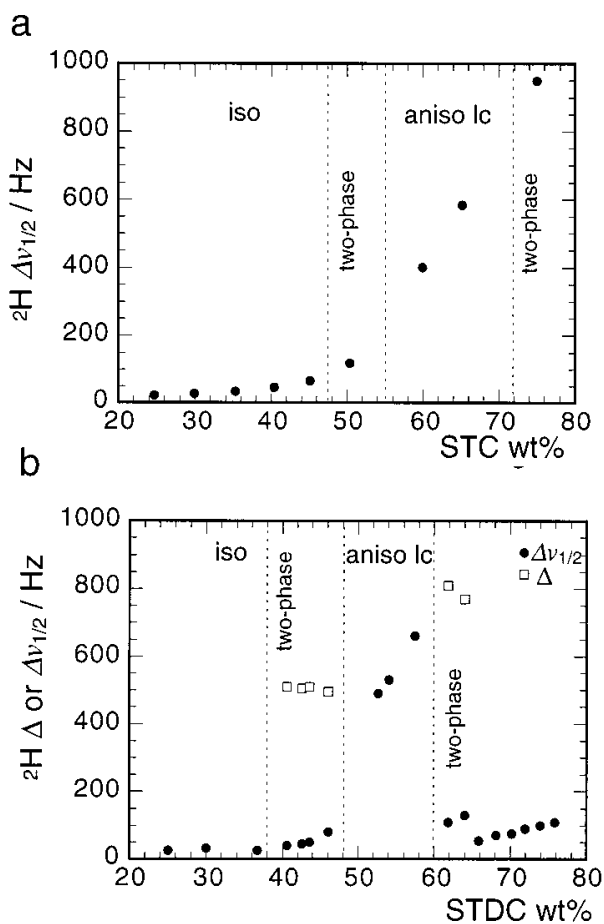

Figure 8. Deuterium splitting $\left({ }^{2} \mathrm{H} \Delta\right)$ or signal half-width $\left({ }^{2} \mathrm{H}\right.$ $\Delta v_{1 / 2}$ ) vs bile salt weight percent for (a) STC, at $18^{\circ} \mathrm{C}$, and (b) STDC, at $12-13^{\circ} \mathrm{C}$.

The ${ }^{2} \mathrm{H}$ quadrupolar splitting for doublets or the halfwidth for single signals are plotted versus surfactant weight percent in parts $a$ and $b$ of Figure 8 for the STC and STCDC systems, respectively. For the STC-water system, no water ${ }^{2} \mathrm{H}$ splitting could be detected in singleor two-phase samples containing the liquid-crystalline phase, even after considerable sample aging (weeks). Samples in the Ic phase showed as broad signals with $\Delta$ values of $0.4-1 \mathrm{kHz}$ (Figure 8a). In the case of STCDC, for samples in the Ic region or in biphasic regions in the vicinity of the Ic phase, the spectra showed as a broad signal plus a small splitting on the order of $0.5-0.8 \mathrm{kHz}$ (Figure 8b).

The ${ }^{2} \mathrm{H}$ NMR splittings are much smaller than those usually observed for typical liquid-crystalline phases in surfactant systems. ${ }^{36,37,30}$ This effect might result from very small values of the order parameter for the binding of water, indicating in turn a small degree of anisotropy for the liquid crystal. The nonappearance of ${ }^{2} \mathrm{H}$ splittings for anisotropic phases has been previously reported. ${ }^{38}$ The broad signal s observed arean indication that fast exchange conditions (between "free" and "bound" water) are not fulfilled, possibly resulting from the fact that the Ic microcrystallites in the sample are not large enough (not even after weeks of undisturbed aging).

C. SAXS Measurements. Structural length scales in lyotropic liquid crystal sareon the order of $10-500 \AA$, and thus, SAXS data can often be used to determine the type of liquid crystal present in a sample. Different liquid crystals (lamellar, hexagonal, cubic, etc.), possessing some type of long-range structural order, show well-defined Bragg peaks (reflections), resulting from the constructive interference of reflected X-rays. Theoretically, a hexagonal phase is built up of infinitely long cylindrical aggregates packed in a 2-D hexagonal array, with Bragg peak

(36) Tiddy, G. J . T. Phys. Rep. 1980, 57, 1

(37) Kang, C.; Khan, A. J. Colloid Interface Sci. 1993, 156, 218.

(38) Khan, A.; J önsson, B.; Wennerström, H.J . Phys. Chem. 1985 89, 5180. 
positions corresponding to

$$
q_{h k}=\frac{2 \pi\left(h^{2}+k^{2}+h k\right)^{1 / 2}}{d}
$$

where $q_{h k}$ is the scattering vector of the [hk] reflection, $h$ and $\mathrm{k}$ are the Miller indices, and $\mathrm{d}$ is the Bragg lattice spacing. In the hexagonal phase, $\left(h^{2}+k^{2}+h k\right)^{1 / 2}$ takes the values $1, \sqrt{ } 3, \sqrt{ } 4, \sqrt{ } 7$, and $\sqrt{ } 9$, corresponding to the first five reflections (hk) = (10), (11), (20), (21), and (30). Thehexagonal cylinder radius $r_{c}$, in thenormal hexagonal phase, can be calculated by

$$
r_{c}=d\left(\frac{2 f}{\pi \sqrt{3}}\right)^{1 / 2}
$$

where $f$ is the volume fraction of the nonpolar domains. The interfacial area per surfactant molecule $a_{s}$, also in the normal hexagonal phase, is obtained according to

$$
a_{s}=\frac{2 f v_{s}}{r_{c} \phi_{s}}
$$

where $\mathrm{v}_{\mathrm{s}}$ is the volume per surfactant molecule and $\phi_{\mathrm{s}}$ is thevolumefraction of surfactant. I n thereversehexagonal phase, f should be replaced with $1-f$ (i.e., the volume fraction of polar domains) in eqs 5 and 6.

In a previous report for the STDC-water system, it was found that samples within the Ic phase showed a diffraction pattern with peaks on the order of 1:1.73:1.89, thus with close similarity to the hexagonal diffraction pattern $(1: \sqrt{ } 3: 2: \sqrt{ } 7: 3)$. In this work, a sample with STC at 60 wt \%, lying in the lc phaseregion, shows a practically unequivocal hexagonal diffraction spectra up to fifth-order reflection, with peaks in the order 1:1.75:2.00:2.77:3.09 (Figure 9a). In other binary systems, patterns very close to a hexagonal onecould beidentified, but only for samples in biphasic regions containing the anisotropic Ic phase. This is the case shown in Figure 9b for STCDC, at 44 wt $\%$, where four correlation peaks in the order 1:1.76:2.00: 2.66 are seen. F or SDC, at a composition of 39.1 wt \% in the close vicinity of the Ic boundary, a pattern with the peak order 1:1.71:2.00:2.69 can also be identified.

All the SAXS data clearly points to a hexagonal-type liquid crystal formed by the bile salts here investigated, entirely consistent with thecharacteristicoptical textures in Figures 4 and 5 . H owever, this phase is most probably built up of aggregates not of a conventional type. It is likely that such aggregates bear a close structural connection with the long rodlike aggregates (or fibers) that have been drawn from concentrated isotropic solutions and for which a helical structure has been pro-

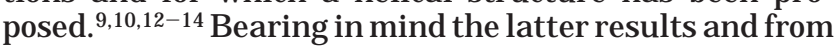
the location of the Ic phase in the phase diagram, we tentatively assign this phasetoa reverse-type of hexagonal phase. Under this assumption, theSAXS results obtained for the representative samples in Figure 9 were analyzed and areshown in Table 1.Thecal culated latticeparameters in Table 1 include the lattice spacing $d$, the lattice parameter $\alpha$ (nearest-neighbor distance, $\alpha=2 d / \sqrt{ } 3$ ) and the hexagonal cylinder radius $r_{c}$. In these calculations, $v_{s}$ $=530 \AA^{3}$ was used, ${ }^{2}$ it was assumed that $\mathrm{f} \approx \phi_{\mathrm{s}}$, and consistently with the proposed reversestructure, thefactor $(1-f)$ was used in eqs 5 and 6 . The structural parameters listed are to betaken on a semiquantitative basis, in view of the approximations made. Nonetheless, they allow us a qualitative fruitful discussion of trends. From Table 1, it can be seen that STC has the smallest cylinder radius and the largest interfacial area per molecule $\left(65 \AA^{2}\right)$. This
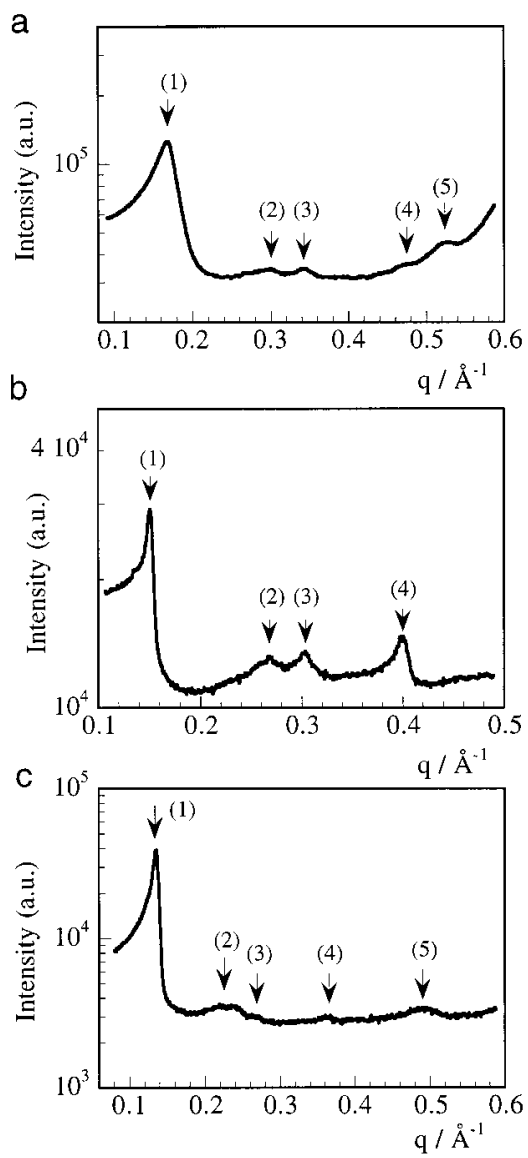

Figure 9. Slit-smeared SAXS patterns for bile salt-water samples: (a) $59.9 \mathrm{wt} \% \mathrm{STC}$, at $22{ }^{\circ} \mathrm{C}$; (b) $43.6 \mathrm{wt} \%$ STCDC, at $15{ }^{\circ} \mathrm{C}$; (c) 39.1 wt $\%$ SDC, at $22{ }^{\circ} \mathrm{C}$. The numbers indicate the nth order correlation peak ascribed to a hexagonal lattice.

is in agreement with the fact that this salt has three hydroxy groups and thus is the least hydrophobic of the series. STCDC has a larger value of $a_{s}$ than STDC and SDC, and this fact can be rationalized as follows: with the two $\mathrm{OH}$ groups in carbons 3 and 7 (cf. Figure 1b), STCDC cannot pack in the interface as tightly as STDC and SDC (OH groups in carbons 3 and 12). The observed trend in $\mathrm{a}_{\mathrm{s}}$, STC > STCDC > SDC > STDC, is thus essentially consistent with the solubility sequence of the salts, as discussed below.

\section{Summary}

It has been shown that all the investigated bile salt mol ecules form an anisotropic liquid-crystall ine phase in water, between the isotropic solution and the hydrated crystals. This pattern of lyotropic behavior appears to be the general case for bile salts. A broad rationalization of the observed phase behavior can be done on the basis of the known solubility of the salts, in turn dependent on their chemical structure. ${ }^{2}$ The trihydroxy salts are more solublethan the dihydroxy ones. Thetaurine and glycine conjugates are much more soluble than the free salts due to their peptide bonds and strong ionic groups, and the taurineis slightly more sol ublethan theglycine due to its higher polarity. The chenodeoxy derivatives are slightly more soluble than the deoxy ones. All these observations are reflected in the obtained cmcs for different salts of the same alkali metal ion. ${ }^{2}$ Accordingly, the maximum solubility for the salts studied here follows the order STC > STCDC > STDC > SGDC > SDC (i.e., the "hydrophilicity" of the salts decreases in this order). From the phase 
Table 1. Results for SAXS Measurements in Liquid-Crystalline Samples of Different Bile Salt-Water Systems

\begin{tabular}{lcccccccccc}
\hline bile salt & wt \% & $\mathrm{d}^{\mathrm{a}}(\AA)$ & $\mathrm{a}^{\mathrm{b}}(\AA)$ & $\mathrm{r}_{\mathrm{c}}{ }^{\mathrm{c}}(\AA)$ & $\mathrm{a}_{\mathrm{s}}{ }^{\mathrm{d}}\left(\AA^{2}\right)$ & $\mathrm{q}_{1}{ }^{\mathrm{e}}(10)\left(\AA^{-1}\right)$ & $\mathrm{q}_{2}(11)\left(\AA^{-1}\right)$ & $\mathrm{q}_{3}(20)\left(\AA^{-1}\right)$ & $\mathrm{q}_{4}(21)\left(\AA^{-1}\right)$ & $\mathrm{q}_{5}(30)\left(\AA^{-1}\right)$ \\
\hline STC & 59.9 & 37.0 & 42.7 & 16.3 & 65.0 & 0.170 & 0.297 & 0.340 & 0.472 & 0.526 \\
STDC & 40.0 & 51.5 & 59.5 & 26.3 & 40.3 & 0.122 & 0.210 & 0.231 & 0.400 \\
STCDC & 43.6 & 41.9 & 48.4 & 20.9 & 50.6 & 0.150 & 0.265 & 0.303 & 0.361
\end{tabular}

a Lattice spacing, obtained from the (10) reflection. ${ }^{b}$ Lattice parameter (nearest-neighbor distance). ${ }^{\mathrm{c}}$ Hexagonal cylinder radius. ${ }^{d}$ Interfacial area per surfactant molecule. ${ }^{e} \mathrm{q}_{\mathrm{n}}$ is the $\mathrm{q}$ value for the $\mathrm{nth}$ order (hk) reflection. ${ }^{\mathrm{f}}$ Values from Edlund et al. ${ }^{26}$

diagrams in Figure 2, it can be seen that, at $20^{\circ} \mathrm{C}$, the solubility order is STC $>$ STCDC $\geq$ SGDC > STDC > SDC, which is not far from theexpected one. For example, at $20^{\circ} \mathrm{C}$, thesolubilities in bilemolar fraction for STC and STDC is 0.036 and 0.011 , respectively, indicating thelarge effect on solubility of the additional $\mathrm{OH}$ group.

The observed thermal stability of the Ic phase for the bile salts is small compared to those of typical ionic surfactants. This fact suggests weak energy differences between the isotropic sol ution and the liquid crystal. The thermal stability of a liquid-crystall ine phase is expected to increase with an increasing hydrophilic character of the surfactant headgroup (for identical hydrophobicity). For instance, phase-behavior studies on sodium and potassium surfactants show a relative hydrophilicity for anionic groups corresponding to carboxylate $\gg$ sulfonate > sulfate. ${ }^{39}$ The comparison between the phase diagrams of SDC, SGDC, and STDC (which differ only in headgroup composition) in Figure 2 does not show significant differences in thermal stability, but the taurine salt (the most hydrophilic) is indeed slightly more stable than the glycine and the free salts. When comparing the mole fraction of bile salt for the lower limit of thelc phase, one has $0.033,0.025$, and 0.019 for SDC, SGDC and STDC, respectively. Moreover, the width of the Ic phase in composition has the order STDC $\gg$ SGDC $\approx$ SDC. Solely on the basis of these observations, it appears that the increasing hydrophilicity of the headgroup favors the formation of the lc phase. The effect on phase behavior of the number and position of $\mathrm{OH}$ groups is considerable, in view of the phase diagrams for the STC, STDC, and STCDC systems. In particular, changing the $\mathrm{OH}$ group from the $\alpha \mathrm{C}-12$ to the $\alpha \mathrm{C}-7$ position on going from the deoxy to the chenodeoxy salt, respectively, brings about a lower thermal and compositional stability for the Ic phase. What appears to be borne out from the phase diagram observations is that chemical structure plays a relatively intricaterolein theaggregation behavior of bile salts. This is not surprising, since both hydrophobi c backto-back interactions and intermolecular hydrogen bonds are proposed to take place in the self-assembly of the molecules.

The kinetics of phase transformation is different for the different bile acid salts. For instance, birefringent structures form in 1-2 days in the STDC-water system, while much longer times, up to months, are required for the other salts. The very long time required to have equilibriumstructures is an indication that the difference in stability between the different states of organization for bileacid salts is small. Someunusual phase-transition phenomena are observed. There is slow formation of anisotropic textures from the corresponding dispersed solids and occurrence, for some salts, of transient spherulitic domains. These uncommon nucleation effects, the

(39) Laughlin, R. G. The Aqueous Phase Behavior of Surfactants; Academic Press: San Diego, 1994. long equilibration times and the puzzling temporal sequence of the transformations, may have led most authors in the past to discard tout court the occurrence of liquid-crystalline phases in such systems.

The optical textures, the SAXS data, and the ${ }^{2} \mathrm{H}$ NMR spectra taken in combi nation point toan ani sotropicliquidcrystalline phase of hexagonal symmetry. This phase is most probably built up of nonconventional micellar-like aggregates of the reverse type. The SAXS spectra were analyzed under this assumption, and some reasonable trends wereidentified. Thepossibility of a closestructural relation between this hexagonal phaseand thelong rodlike aggregates of helical structure, previously described for the isotropic phase, ${ }^{12-14}$ has to be kept in mind. The solutions observed at concentrations of bileacid salt higher than those of the Ic phase are extremely viscous, and it is not easy to ascertain whether they are true solutions or cubic phases. A more detailed study of this viscous isotropic phase is required.

Lyotropic liquid crystals formed by surfactants such as soaps, detergents, and lipids, are consider ed to require a liquidlike state of the flexible chains as a prerequisite for their assembly. Obviously this does not hol d for molecules with rigid ring structures which nonethel ess bear lyotropic properties. There is indeed a vast class of molecules, termed chromonic materials and including drugs, dyes, and antibiotics, which show the occurrence of lyotropic liquid crystals such as nematic and hexagonal phases (cf. recent review by Lydon ${ }^{40}$ ). Such molecules do not have surface activity, they do not possess flexible aliphatic chains since they are usually based on aromatic ring structures, and they often have polar groups around the peripheries of thering structure (implying that polar and apolar regions are not clearly separated). There is thus some structural analogy between the bile salts and such molecules. Concomitantly, in terms of lyotropic behavior, bile salts can be seen as lying in an intermediate position between thechromonic materials and thecommon flexible chain surfactants. It must be borne in mind that liquidcrystal formation does not necessarily imply any particular state of the molecule but rather a positional order of domains. I $n$ this context, it is not at all surprising that the bile acid salts, which form supramolecular aggregates of certain compl exity in solution (viz. fiberlike structures), can also form liquid-crystalline phases.

Acknowledgment. TheSwedish Research Council for Engineering Sciences (TFR) and PRAXIS XXI, Portugal (Project 2/2.1/QUI/411/94) are gratefully acknowledged for financial support. H.E. gratefully acknowledges STINT (The Swedish F oundation for I nternational Cooperation in Research and Higher Education), for a Postdoctoral Scholarship in theDepartment of Chemical Engineering, University of Delaware.

LA9912803

(40) Lydon, J . Curr. Opin. Colloid Interface Sci. 1998, 3, 455. 